\title{
Imaging Immunometabolism in Atherosclerosis
}

\author{
Philip Z. Mannes ${ }^{1}$ and Sina Tavakoli ${ }^{1-3}$ \\ ${ }^{1}$ Department of Radiology, University of Pittsburgh, Pittsburgh, Pennsylvania; ${ }^{2}$ Department of Medicine, University of Pittsburgh, \\ Pittsburgh, Pennsylvania; and ${ }^{3}$ Heart, Lung, Blood, and Vascular Medicine Institute, University of Pittsburgh Medical Center, \\ Pittsburgh, Pennsylvania
}

\begin{abstract}
Over the past decade, there has been a growing recognition of the links between intracellular metabolism and immune cell activation, that is, immunometabolism, and its consequences in atherogenesis. However, most immunometabolic investigations have been conducted in cultured cells through pharmacologic or genetic manipulations of selected immunologic or metabolic pathways, limiting their extrapolation to the complex microenvironment of plaques. In vivo metabolic imaging is ideally situated to address this gap and to determine the clinical implications of immunometabolic alterations for diagnosis and management of patients. Indeed, ${ }^{18} \mathrm{~F}$-FDG has been widely used in clinical studies with promising results for risk stratification of atherosclerosis and monitoring the response to therapeutic interventions, though the biologic basis of its uptake in plaques has been evolving. Herein, we describe recent advances in understanding of immunometabolism of atherosclerosis with an emphasis on macrophages, and we review promising metabolic imaging approaches using ${ }^{18} \mathrm{~F}-\mathrm{FDG}$ and other PET radiotracers.
\end{abstract}

Key Words: molecular imaging; atherosclerosis; immunometabolism; inflammation; macrophage; metabolic imaging

J Nucl Med 2021; 62:896-902

DOI: 10.2967/jnumed.120.245407

$\mathbf{T}$ hrough determining the severity of luminal stenosis and compromised blood flow, conventional imaging, such as angiography and perfusion imaging, has revolutionized the diagnosis and management of flow-limiting atherosclerotic plaques causing chronic ischemia (1). However, these approaches are limited for identification of lesions at risk of acute complications (e.g., plaque rupture or erosion) leading to acute myocardial infarction or other thromboembolic events, as well as for assessment of the response to therapeutic interventions (1). Imaging pathophysiologic processes occurring within the plaques, rather than their late structural or physiological sequelae on luminal patency or perfusion, promotes a precision approach to atherosclerosis by improving the risk stratification of patients and allowing for early monitoring of disease progression or response to therapy (1).

Inflammation as a key pathogenic driver of atherosclerosis has been a major target for molecular imaging (1), a role that has been further highlighted with the emergence of novel immunomodulatory interventions. The versatility of PET to provide insights into various metabolic pathways, along with the growing discoveries in

Received Sep. 24, 2020; revision accepted Jan. 27, 2021.

For correspondence or reprints, contact Sina Tavakoli (sit23@pitt.edu). Published online May 7, 2021.

COPYRIGHT (C) 2021 by the Society of Nuclear Medicine and Molecular Imaging. immunometabolism, defined as the interplay between cell metabolism and immune response, have placed it at the forefront of molecular imaging of atherosclerosis.

Here, we will review recent immunometabolic discoveries in atherosclerosis and discuss promising strategies to noninvasively image plaque immunometabolism.

\section{INFLAMMATION AND ATHEROGENESIS}

Atherosclerosis is a chronic inflammatory disease of the arterial wall, which starts by subendothelial deposition of lipoproteins in areas of perturbed blood flow and endothelial activation (2). Enzymatic and nonenzymatic modifications of the deposited lipoproteins generate proinflammatory derivatives that trigger innate and adaptive immune responses, resulting in recruitment of leukocytes and migration or proliferation of resident cells within the vessel wall, for example, vascular smooth muscle cells (VSMCs), leading to plaque expansion $(1,2)$. The balance between proinflammatory and inflammation-resolving processes within the plaque determines if a lesion stabilizes/regresses or further expands, eventually forming a core largely composed of lipids and necrotic cells $(1,2)$. Formation of a fibrous cap rich in collagen and VSMCs is another critical process, which contributes to plaque stability.

Vulnerable plaques, characterized by thin fibrous caps, large necrotic cores, and abundant inflammatory cells, are prone to fibrous cap rupture and have been historically the most common cause of acute complications of atherosclerosis (3). However, improved control of cardiovascular risk factors, particularly hyperlipidemia, over the past few decades has led to significant declines in the prevalence of rupture-prone plaques, with concomitant increases in the prevalence of endothelial erosion as the cause of acute complications (3). These plaques often contain smaller necrotic cores and fewer inflammatory cells. Therefore, imaging strategies to identify erosion-prone from rupture-prone plaques may be distinct.

\section{IMMUNOMETABOLISM AND ATHEROSCLEROSIS}

Metabolic adaptations are critical for leukocytes to alter their functions in response to various proinflammatory and inflammationresolving stimuli. However, immunometabolism has been largely studied ex vivo using metabolic tracing in conjunction with pharmacologic or genetic manipulations of specific metabolic and immunologic pathways, which do not recapitulate the complexities of the plaque microenvironment. Metabolic imaging is well positioned to bridge this gap and to translate immunometabolic discoveries into clinical applications.

In this review, we will focus on macrophages as the most abundant leukocytes in plaques with critical functions throughout different stages of atherogenesis (4). Despite the contribution of other 
immune cells, including neutrophils, dendritic cells, and lymphocytes, to atherogenesis (2), these cells are present in smaller numbers than are macrophages (and other resident cells within the vessel wall, such as VSMCs), thus challenging their metabolic assessment by PET.

Macrophage metabolism has been largely studied in the context of M1 (proinflammatory) and M2 (inflammation-resolving) polarization states, representing the extreme ends of the functional diversity of macrophages in response to ex vivo activation with selected proinflammatory (e.g., interferon- $\gamma[\mathrm{IFN}-\gamma]$ or lipopolysaccharide) or antiinflammatory (e.g., interleukin [IL]-4) stimuli. However, this dichotomization is not a physiologic phenomenon, and development of less reductive approaches is required to delineate the immunometabolic heterogeneity of macrophages in atherosclerosis. Within this constraint, observational studies suggest that macrophages resembling M1 versus M2 polarization states have opposing effects in atherogenesis and contribute to plaque progression/vulnerability versus regression/stability, respectively (5).

\section{M1 Polarization}

Metabolic reprogramming of M1 polarized macrophages is mostly studied after activation with lipopolysaccharide, as a classic pathogen-derived stimulus (4). Enhanced glucose uptake and glycolysis along with a suppressed tricarboxylic acid (TCA) cycle and reduced oxidative phosphorylation (OxPhos) are hallmarks of lipopolysaccharide-stimulated macrophages $(4,6)$. Activation of hypoxia-inducible factor- $1 \alpha$ (HIF- $1 \alpha)$ is a key regulatory mechanism of lipopolysaccharide-induced metabolic reprogramming, which mimics a pseudohypoxic state manifested by increased expression of glucose transporters and critical rate-limiting glycolytic enzymes, including hexokinases (4). Moreover, lipopolysaccharide causes functional breaks in the TCA cycle by suppressing isocitrate dehydrogenase and succinate dehydrogenase activities (6). These breaks lead to the accumulation of citrate and succinate, which contribute to other immunometabolic changes of macrophages, including increased de novo synthesis of fatty acids, suppression of OxPhos and the electron transport chain, stabilization of HIF- $1 \alpha$, and production of inflammatory mediators, such as IL-1 $\beta$ (4). Upregulation of the oxidative phase of the pentose phosphate pathway is another key metabolic feature of lipopolysaccharide stimulation, which provides macrophages with the 5-carbon sugars and nicotinamide adenine dinucleotide phosphate needed for the biosynthesis of fatty acids and sterols, and redox regulation (4).

Emerging evidence supports the role of HIF- $1 \alpha$-mediated metabolic reprogramming in atherogenesis. Notably, myeloid cell expression of HIF- $1 \alpha$ is critical in high-fat-diet-induced atherosclerosis and development of the necrotic core in a mouse model (7). Additionally, hematopoietic deficiency of glucose transporter 1 reduces both monocyte influx into plaques and progression of atherosclerosis $(8)$.

\section{M2 Polarization}

The conventional M2 polarizing stimulus IL-4 induces a markedly different metabolic signature, compared with that of lipopolysaccharide, and is regulated through different transcriptional machinery, including Akt, STAT-6, and PGC1- $\beta$ (4). Moreover, IL-4-induced metabolic changes are relatively delayed and require mitochondrial biogenesis, a key driver of enhanced OxPhos, TCA cycle activity, and fatty acid oxidation (4). Therefore, mitochondrial damage and compromised respiration present in atherosclerotic plaques may underlie the impaired inflammation-resolving capacity of plaque macrophages, particularly in the fibrous cap and necrotic core (9). Interestingly, restoration of plaques' mitochondrial respiration leads to smaller necrotic cores and larger fibrous caps, supporting the plaque-stabilizing role of mitochondrial respiration (9).

It should, however, be noted that metabolic reprogramming of macrophages is stimulus-specific and that various proinflammatory (e.g., oxidized phospholipids and granulocyte-macrophage colony-stimulating factor [GM-CSF]) and antiinflammatory (e.g., macrophage colony-stimulating factor [M-CSF]) stimuli result in overlapping metabolic alterations (Fig. 1A). Moreover, the metabolic profiles of additional plaque-associated macrophage phenotypes outside the M1 and M2 dichotomy, including Mox and Mhem (5), have not been well established.

\section{IMMUNOMETABOLIC IMAGING}

In the following sections, promising PET tracers for imaging the immunometabolism of atherosclerosis are briefly reviewed (Fig. 1B; Table 1).

\section{${ }^{18}$ F-FDG}

After the initial retrospective reports of ${ }^{18} \mathrm{~F}$-FDG uptake in atherosclerosis, several studies demonstrated increased ${ }^{18} \mathrm{~F}-\mathrm{FDG}$ uptake in plaques with features of vulnerability (e.g., large lipid core and intraplaque hemorrhage) (10) and in symptomatic patients (e.g., recent transient ischemic attack or stroke), compared with stable/asymptomatic lesions (11). Further investigations revealed that plaques' ${ }^{18} \mathrm{~F}-\mathrm{FDG}$ uptake correlates with their inflammatory burden, particularly macrophage content, and can be reproducibly quantified (12), suggesting the potential of ${ }^{18} \mathrm{~F}-\mathrm{FDG}$ PET for noninvasive monitoring of plaque inflammation and risk of vulnerability. Moreover, ${ }^{18}$ F-FDG uptake in large (e.g., aortic, carotid, and iliofemoral) arteries was shown to correlate with various cardiovascular risk factors and improve the prediction of future cardiovascular events, including stroke and myocardial infarction, beyond conventional risk stratification tools, such as Framingham risk score and severity of carotid artery stenosis $(13,14)$. A dose-dependent decline in the ${ }^{18}$ F-FDG uptake of the aortic and carotid arteries was also shown as early as 4 wk after treatment with atorvastatin (15), indicating its role in monitoring the therapeutic response. However, subsequent studies with novel lipid-lowering or antiinflammatory drugs showed no significant decline in arterial uptake of ${ }^{18} \mathrm{~F}$-FDG (16), indicating that the role of ${ }^{18} \mathrm{~F}$-FDG in monitoring the therapeutic response needs to be validated with individual drugs.

Despite extensive clinical investigations, the biologic basis of ${ }^{18}$ F-FDG uptake in atherosclerosis has been evolving. Although ${ }^{18}$ F-FDG uptake was largely attributed to its enhanced uptake by proinflammatory macrophages, recent evidence supports that other components of plaques, particularly VSMCs, may have similar or even higher levels of ${ }^{18} \mathrm{~F}$-FDG uptake $(17,18)$. Moreover, increasing evidence supports that ${ }^{18} \mathrm{~F}-\mathrm{FDG}$ uptake, as a stand-alone marker, fails to discriminate the metabolic reprogramming of macrophages in response to different proinflammatory and inflammation-resolving stimuli (19-21). For example, enhanced glucose uptake is not a ubiquitous feature of proinflammatory macrophages and may be absent in macrophages stimulated with IFN- $\gamma$ and TNF- $\alpha$. Additionally, inflammation-resolving stimuli, such as M-CSF (20), and other microenvironmental factors, for 


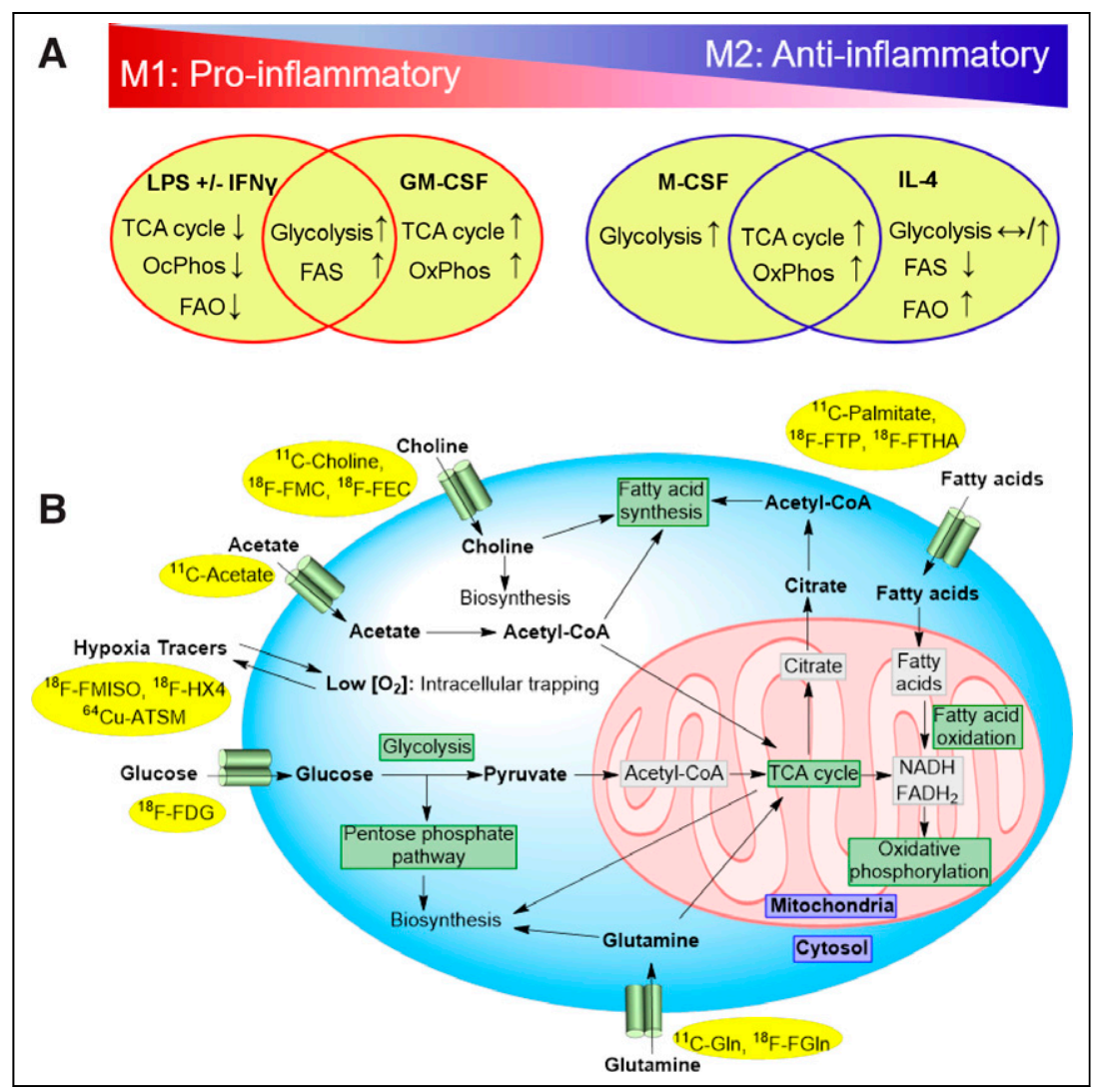

FIGURE 1. (A) Overlapping and stimulus-specific metabolic reprogramming of macrophages polarized into proinflammatory (M1) and inflammation-resolving (M2) states. Metabolic changes in response to lipopolysaccharide with or without IFN- $\gamma$ and GM-CSF, as examples of M1-polarizing stimuli, vs. IL-4 and M-CSF, as examples of M2-polarizing stimuli, are summarized. Immunometabolic profiles of macrophages within plaque microenvironment are far more complex and cannot be accurately extrapolated from M1/M2 polarization scheme. (B) Major metabolic pathways targeted by PET imaging and examples of available radiotracers. ${ }^{64} \mathrm{Cu}-\mathrm{ATSM}=$ ${ }^{64} \mathrm{Cu}$-copper(II)diacetyl-di(N4-methylthiosemicarbazone); $\mathrm{CoA}=$ coenzyme $\mathrm{A} ; \mathrm{FADH} 2$ = reduced flavin adenine dinucleotide; $F A O=$ fatty acid oxidation; FAS $=$ fatty acid synthesis; ${ }^{18} \mathrm{~F}-\mathrm{FEC}=$ ${ }^{18} \mathrm{~F}$-fluoroethylcholine; ${ }^{18} \mathrm{~F}$-FGln $={ }^{18} \mathrm{~F}$-(2S,4R)-4-fluoro-L-glutamine; ${ }^{18} \mathrm{~F}-\mathrm{FMC}={ }^{18} \mathrm{~F}$-fluoromethylcholine; ${ }^{18} \mathrm{~F}$-FTHA $={ }^{18} \mathrm{~F}$-fluoro-6-thia-heptadecanoic acid; ${ }^{18} \mathrm{~F}$-FTP $={ }^{18} \mathrm{~F}$-fluoro-4-thia-palmitate; ${ }^{11} \mathrm{C}-\mathrm{Gln}=\mathrm{L}-5-{ }^{11} \mathrm{C}$-glutamine; LPS = lipopolysaccharide; $\mathrm{NADH}=$ reduced nicotinamide adenine dinucleotide; OxPhos $=$ oxidative phosphorylation.

example, hypoxia (21) and oxidized phospholipids (22), increase ${ }^{18} \mathrm{~F}-\mathrm{FDG}$ uptake in macrophages and atherosclerotic plaques.

\section{Hypoxia}

Intraplaque hypoxia, particularly in advanced lesions, results from excessive oxygen consumption by metabolically active cells and insufficient oxygen delivery from the lumen and vasa vasorum (7). Hypoxia contributes to the advancement and vulnerability of plaque through expansion of the necrotic core, impairment of efferocytosis, and accentuation of inflammation (4,7,23). Moreover, hypoxia induces the expression of HIF- $1 \alpha$. which is essential for macrophage migration $(24,25)$ and shifts their metabolism toward glycolysis, allowing for the buildup of TCA intermediates necessary to support a proinflammatory response, for example, production of IL-1 $\beta(4,23)$.

Two major classes of hypoxia imaging agents, including nitroimidazole (e.g., ${ }^{18} \mathrm{~F}$-fluoromisonidazole [FMISO] and ${ }^{18} \mathrm{~F}$-(3-fluoro2-\{4-[(2-nitro-1H-imidazol-1-yl)methyl]-1H-1,2,3-triazol-1-yl $\}$ propan-1-ol) $\left.\left[{ }^{18} \mathrm{~F}-\mathrm{HX} 4\right]\right)$ and copper complexed with diacetylbis(N4-methylthiosemicarbazone) analogs, have been used to image atherosclerosis in small clinical studies. Localized ${ }^{18} \mathrm{~F}-\mathrm{HX} 4$ uptake in symptomatic carotid plaques was shown to correlate with arterial wall thickness and ${ }^{18}$ F-FDG uptake (26). ${ }^{18}$ F-FMISO uptake was also found to be increased in symptomatic, compared with the contralateral, carotid plaques in patients with a recent stroke or transient ischemic attack (27). Histologic analysis of endarterectomy specimens trended toward a positive correlation between ${ }^{18}$ F-FMISO and macrophage content but not with HIF- $1 \alpha$ expression or VSMC content (27). Both studies found positive correlations between the uptake of ${ }^{18} \mathrm{~F}$-FDG and that of either ${ }^{18} \mathrm{~F}-\mathrm{HX} 4$ or ${ }^{18} \mathrm{~F}-\mathrm{FMISO}$ (Fig. 2A) $(26,27)$, as is consistent with the major role of hypoxia in promoting glycolysis (21). Encouragingly, a recent paper using ${ }^{64} \mathrm{Cu}$-diacetyl-bis(N4-methylthiosemicarbazone) in asymptomatic patients with carotid stenosis of more than $50 \%$ reported that HIF$1 \alpha$ expression colocalized with $\mathrm{CD}^{+} 8^{+}$ expressing macrophages in PET-positive plaques (28).

One limitation of hypoxia radiotracers is that barriers to diffusion, such as poor vascularization and fibrosis, reduce tracer uptake in hypoxic tissue, likely contributing to the modest target-to-background signal observed with these agents in atherosclerosis. Future work is required to determine whether imaging hypoxia allows for prospective identification of plaques at risk of acute complications or response to medical interventions.

\section{Acetate}

Acetate, a 2-carbon intermediate used in energy-generating and biosynthetic pathways, is transported into cells by monocarboxylate transporters (29) and is converted to acetyl coenzyme A by cytosolic or mitochondrial acetyl coenzyme A synthases (29). Cytosolic acetyl coenzyme A is used for fatty acid synthesis and protein acetylation. Mitochondrial acetyl coenzyme A enters the TCA cycle by condensation with oxaloacetate to form citrate, which can be used to generate adenosine triphosphate through OxPhos or exported to the cytoplasm for fatty acid synthesis, intimately linking acetate and fatty acid metabolism. Acetate metabolism is therefore an attractive imaging target that provides insight into cellular catabolism and anabolism.

The feasibility of imaging ${ }^{11} \mathrm{C}$-acetate uptake by plaques along the large (e.g., aortic, iliac, and carotid) arteries has been shown in patients undergoing PET/CT for oncologic indications (Fig. 2B) (30). High-resolution autoradiography of murine brachiocephalic arteries has also demonstrated that arterial uptake of acetate is localized primarily to macrophage-rich regions of plaques (31). Moreover, it was shown that IFN- $\gamma$ plus lipopolysaccharide markedly suppressed ex vivo acetate uptake by murine macrophages and human carotid endarterectomy specimens, whereas IL-4 
TABLE 1

Summary of Metabolic Radiotracers Used for PET/CT Imaging of Atherosclerosis

\begin{tabular}{|c|c|c|c|c|}
\hline Tracer & Metabolic targets & Advantages and key findings & Limitations & Study \\
\hline${ }^{18} \mathrm{~F}-\mathrm{FDG}$ & $\begin{array}{l}\text { Glucose transport and } \\
\text { phosphorylation }\end{array}$ & $\begin{array}{l}\text { Tracer is readily available and } \\
\text { the most extensively validated } \\
\text { in preclinical and clinical } \\
\text { studies; uptake correlates } \\
\text { with overall inflammatory } \\
\text { burden of plaques, for } \\
\text { example, macrophage } \\
\text { content; early detection of } \\
\text { response to statins is possible }\end{array}$ & $\begin{array}{l}\text { Tracer targets nearly } \\
\text { ubiquitous metabolic } \\
\text { process, with limited } \\
\text { specificity for individual cell } \\
\text { type or phenotype }\end{array}$ & $(12-15)$ \\
\hline${ }^{18} \mathrm{~F}-\mathrm{FMISO}$ & Cellular hypoxia & $\begin{array}{l}\text { Uptake is higher in symptomatic } \\
\text { carotid plaques; positive } \\
\text { correlation exists between } \\
{ }^{18} \mathrm{~F}-\mathrm{FMISO} \text { and }{ }^{18} \mathrm{~F} \text {-FDG } \\
\text { uptake }\end{array}$ & $\begin{array}{l}\text { Diffusion barrier limits uptake; } \\
\text { cell specificity is lacking }\end{array}$ & $(27)$ \\
\hline${ }^{18} \mathrm{~F}-\mathrm{HX} 4$ & Cellular hypoxia & $\begin{array}{l}\text { Positive correlation exists } \\
\text { between }{ }^{18} \mathrm{~F}-\mathrm{HX} 4 \text { and }{ }^{18} \mathrm{~F}- \\
\text { FDG uptake }\end{array}$ & $\begin{array}{l}\text { Diffusion barrier limits uptake; } \\
\text { cell specificity is lacking }\end{array}$ & $(26)$ \\
\hline${ }^{64} \mathrm{Cu}-\mathrm{ATSM}$ & Cellular hypoxia & $\begin{array}{l}\text { Uptake correlates with plaque } \\
\text { hypoxia and macrophage } \\
\text { content; hypoxic cell uptake is } \\
\text { higher than for }{ }^{18} \mathrm{~F}-\mathrm{FMISO} \\
\text { and washout from normoxic } \\
\text { tissues is faster }\end{array}$ & $\begin{array}{l}\text { Diffusion barrier limits uptake; } \\
\text { cell specificity is lacking }\end{array}$ & $(28)$ \\
\hline${ }^{11} \mathrm{C}$-acetate & TCA, FAO, FAS, OxPhos & $\begin{array}{l}\text { Focal uptake occurs in calcified } \\
\text { plaques and arterial segments } \\
\text { without calcifications }\end{array}$ & $\begin{array}{l}\text { Half-life is short, limiting } \\
\text { availability }\end{array}$ & (30) \\
\hline${ }^{11} \mathrm{C}$-choline & FAS, biosynthesis & $\begin{array}{l}\text { Uptake is mostly in arterial } \\
\text { segments with thickening and } \\
\text { increased lipid content; } \\
\text { cardiac uptake is lower than } \\
\text { that of }{ }^{18} \mathrm{~F}-\mathrm{FDG}\end{array}$ & $\begin{array}{l}\text { Half-life is short, limiting } \\
\text { availability }\end{array}$ & $(37)$ \\
\hline${ }^{18} \mathrm{~F}-\mathrm{FMC}$ & FAS, biosynthesis & $\begin{array}{l}\text { Uptake is mostly in arterial } \\
\text { segments with thickening and } \\
\text { increased lipid content }\end{array}$ & $\begin{array}{l}\text { Tracer may be substrate for } \\
\text { distinct set of transporters } \\
\text { from those used to } \\
\text { transport choline }\end{array}$ & $(36)$ \\
\hline${ }^{18} \mathrm{~F}-\mathrm{FEC}$ & FAS, biosynthesis & $\begin{array}{l}\text { Uptake correlates with } \\
\text { cardiovascular risk factors; no } \\
\text { association exists between } \\
\text { uptake and prior } \\
\text { cerebrovascular or } \\
\text { cardiovascular events }\end{array}$ & $\begin{array}{l}\text { Tracer may be substrate for } \\
\text { distinct set of transporters } \\
\text { from those used to } \\
\text { transport choline }\end{array}$ & (38) \\
\hline
\end{tabular}

increased its uptake (31), suggesting a potential role of ${ }^{11} \mathrm{C}$-acetate PET in distinction of proinflammatory and inflammation-resolving macrophages. However, the role of ${ }^{11} \mathrm{C}$-acetate imaging in risk stratification of patients or monitoring the response to antiinflammatory interventions remains to be further explored in clinical studies. Moreover, despite the promise of ${ }^{11} \mathrm{C}$-acetate, less attention has been given to plaque imaging using tracers derived from longer-chain fatty acids.

\section{Choline}

Choline is a quaternary amine important for the biosynthesis of membrane lipids and acetylcholine and for hepatic 1-carbon metabolism. Several choline transport systems have been identified, with choline transporter-like proteins and organic cation transporters being widely expressed (32). Once in the cell, choline and choline- based radiotracers are incorporated into lipids (32), like phosphatidylcholine, which is a major constituent of the outer cell membrane.

Choline has thus emerged as a promising marker of cells with high rates of phospholipid biosynthesis, including proliferating cells. Lipopolysaccharide stimulation of macrophages increases choline transporter-like protein 1-mediated transport of choline, which is required for their proinflammatory activation $(33,34)$. Reducing choline uptake through blocking choline transporter-like protein 1 or culturing in choline-deficient medium impairs macrophage activation, alters the secretion of inflammatory cytokines, and inhibits the residual mitochondrial activity $(33,34)$. Multiple PET radiotracers, including ${ }^{11} \mathrm{C}$-choline, ${ }^{18} \mathrm{~F}$-fluorocholine, and ${ }^{18} \mathrm{~F}$-fluoroethylcholine, have been developed (Fig. 2C), though the fluorocholine-based radiotracers may be substrates for a distinct set of transporters $(32,35)$. 


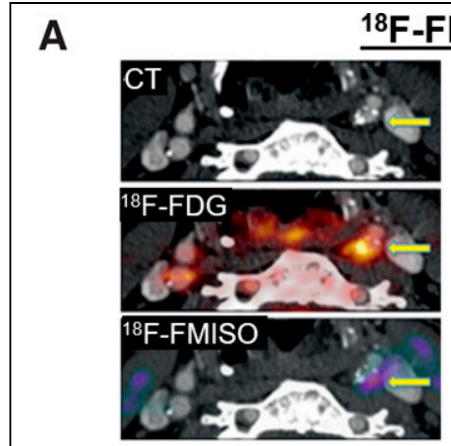

${ }^{18} \mathrm{~F}-\mathrm{FMISO}$ vs. ${ }^{18} \mathrm{~F}-\mathrm{FDG}$

B
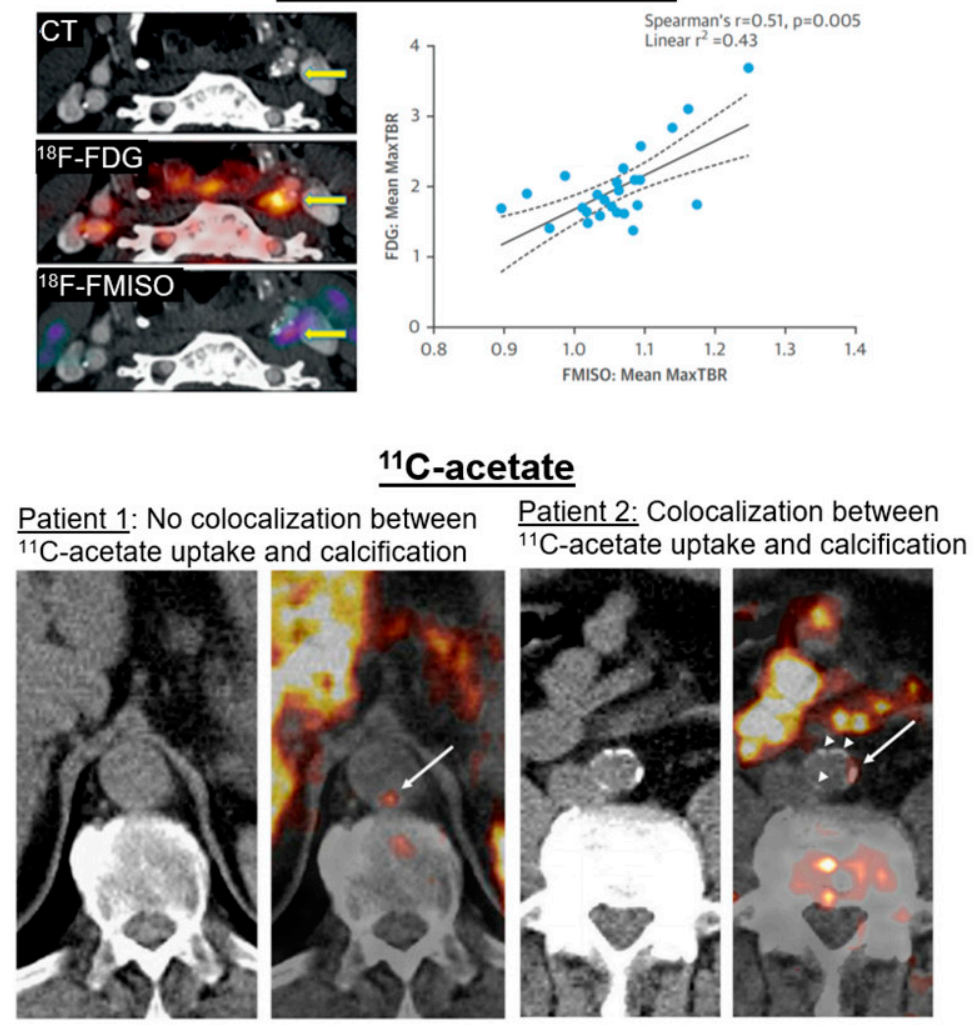

C

11C-choline

Patient 1: No colocalization between ${ }^{11} \mathrm{C}$-choline uptake and calcification
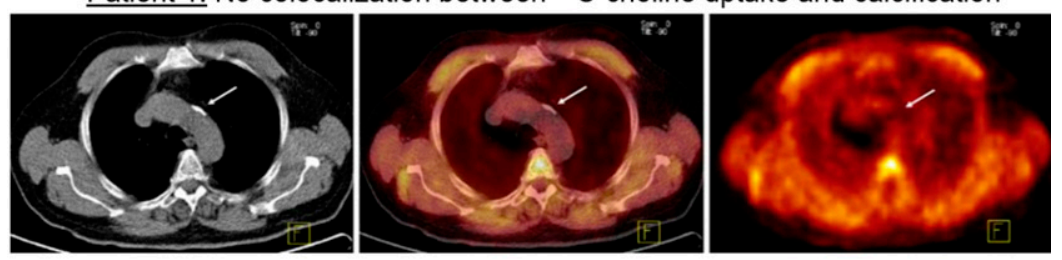

Patient 2: Colocalization between ${ }^{11} \mathrm{C}$-choline uptake and calcification
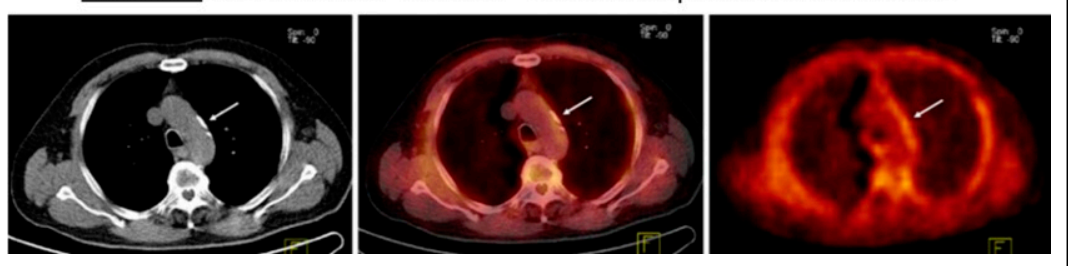

FIGURE 2. PET metabolic imaging of atherosclerosis. (A) Examples of ${ }^{18} \mathrm{~F}-\mathrm{FMISO}$ and ${ }^{18} \mathrm{~F}-\mathrm{FDG}$ PET/CT in patient with carotid artery atherosclerosis demonstrate uptake of both tracers in left carotid artery plaque (arrows). Graph shows that ${ }^{18} \mathrm{~F}$-FMISO uptake correlates with ${ }^{18} \mathrm{~F}$-FDG uptake. (B) Examples of ${ }^{11} \mathrm{C}$-acetate PET/CT of aorta in patients receiving whole-body scans for oncologic indications. Images from patient 1 indicate ${ }^{11} \mathrm{C}$-acetate uptake in aortic region without calcified plaques, whereas images from patient 2 demonstrate colocalization of ${ }^{11} \mathrm{C}$-acetate uptake with aortic calcifications (arrows point to areas of arterial ${ }^{11} \mathrm{C}$-acetate uptake). (C) Examples of ${ }^{11} \mathrm{C}$-choline PET/CT of aortic arch in men receiving whole-body scans for assessment of prostate cancer. Colocalization of ${ }^{11} \mathrm{C}$-choline uptake with aortic calcifications is observed in patient 2 but not in patient 1 (arrows indicate areas of aortic arch calcification). MaxTBR $=$ maximum target-to-background ratio. (Reprinted with permission of $(27,30,37)$.)

The feasibility of choline-derived radiotracers for imaging calcified and noncalcified plaques within the aortic, iliac, and carotid arteries was shown in men undergoing ${ }^{18} \mathrm{~F}$-fluoromethylcholine,
${ }^{11} \mathrm{C}$-choline, and ${ }^{18} \mathrm{~F}$-fluoroethylcholine PET for prostate cancer (36-38). Additionally, plaques' uptake of ${ }^{18} \mathrm{~F}$-fluoromethylcholine strongly correlates with their macrophage content in patients with severe carotid stenosis (39). These early choline-based imaging studies are promising, although additional studies are needed to examine the association between choline uptake and various macrophage activation states or plaques' risk of vulnerability.

\section{Glutamine}

Glutamine is an attractive imaging target given its essential roles in major biosynthetic and metabolic pathways (6). For instance, glutamine replenishes TCA cycle intermediates, by conversion to glutamate and $\alpha$-ketoglutarate, which serve as carbon sources for glucose, fatty acid, and protein synthesis. Additionally, glutamine metabolism is used for nicotinamide adenine dinucleotide phosphate (NADP) production, required for nitric oxide production, fatty acid synthesis, and glutathione regeneration (6). Glutamine also serves as a nitrogen source for purine and pyrimidine synthesis.

Macrophages have high levels of glutamine use (6). Alterations in glutamine metabolism influence macrophage function in both proinflammatory and inflammation-resolving states. Glutamine metabolism is required for IL- $1 \beta$ production by lipopolysaccharide-stimulated macrophages (23). This proinflammatory function is driven through glutamine entering a functionally broken TCA cycle and contributing to increased intracellular succinate levels and HIF-1 $\alpha$ stabilization (40). In contrast, IL-4 causes glutamine shuttling into a functional TCA cycle, which reinforces an M2-like macrophage phenotype through epigenetic modification of M2-specific gene promoters, increased OxPhos and fatty acid oxidation, and destabilization of HIF-1 $\alpha$ through an increased $\alpha$-ketoglutarate-to-succinate ratio $(24,40)$. Interestingly, hyperinflammatory macrophages stimulated with lipopolysaccharide and oxidized phospholipids use glutamine along with mitochondrial respiration to support IL-1 $\beta$ production (22).

Distinct patterns of ${ }^{18} \mathrm{~F}$-FDG and ${ }^{14} \mathrm{C}$-glutamine uptake by aortic atherosclerotic plaques have been shown using high-resolution autoradiography in a mouse model of atherosclerosis, suggesting that combined imaging of glutamine and glucose metabolism may better delineate the immunometabolic heterogeneity of plaques (19). Consistently, combined measurement of glucose and glutamine uptake improves the identification of different activation states of macrophages induced by conventional polarizing agents (19). 
Despite the availability of glutamine-derived PET radiotracers, such as ${ }^{18} \mathrm{~F}-(2 S, 4 R)-4$-fluorogluta mine and ${ }^{11} \mathrm{C}$-glutamine, the feasibility of in vivo imaging of glutamine metabolism in atherosclerosis has remained to be determined.

\section{LIMITATIONS}

Despite the unique role of PET to delineate metabolism in vivo, the immunometabolic complexity of plaques is incompletely captured using individual metabolic substrates because the targeted pathways are exploited by a variety of cells, sometimes nearubiquitously. Moreover, available radiotracers primarily assess the first steps of metabolism, that is, membrane transport or intracellular trapping, and cannot discern the downstream divergence of intracellular use of substrates. Overlapping metabolic signatures of activated immune cells further complicate the assessment of inflammatory states of plaques using a single metabolic pathway. Therefore, unraveling the biologic correlates of radiotracer uptake in atherosclerosis requires meticulous correlations of highresolution PET, autoradiography, and histologic profiling of plaques' immune cells. Moreover, current metabolic imaging approaches to atherosclerosis are focused mostly on detection of rupture-prone plaques and may be ineffective for identification of plaques at risk of erosion.

\section{FUTURE DIRECTIONS}

Recent state-of-the-art immunometabolic discoveries have advanced our understanding of the biologic basis of ${ }^{18} \mathrm{~F}-\mathrm{FDG}$ uptake in atherosclerosis and highlighted the promise of imaging other metabolic pathways, described herein, to improve the noninvasive characterization of plaque inflammation. However, there is a dire need to transition toward in vivo immunometabolic phenotyping of atherosclerosis. Combined imaging of two or more metabolic targets/pathways is a direction that improves the distinction of different inflammatory states of immune cells (19) and may partially address the limited cell-specificity of metabolic radiotracers and the overlapping metabolic signatures of different activation states of immune cells (and other cells residing in the inflamed tissues). However, this approach is logistically complex in clinical settings. Alternatively, development of novel metabolic imaging tracers that are simultaneously targeted toward, or delivered to, specific cell types may address this limitation.

A multidimensional approach extending from preclinical investigations to large-scale longitudinal clinical cohort studies and clinical trials is required to establish the role of immunometabolic imaging as a precision medicine tool in noninvasive plaque characterization and prediction of atherosclerosis outcomes using clinically established endpoints (e.g., acute coronary syndrome, embolic stroke, and progressive stenosis or calcification) or in monitoring the response to therapeutic interventions.

\section{DISCLOSURE}

Sina Tavakoli is supported by NIH-NHLBI K08-HL144911 and NIH-NIBIB R21-EB027871. No other potential conflict of interest relevant to this article was reported.

\section{REFERENCES}

1. Tavakoli S, Vashist A, Sadeghi MM. Molecular imaging of plaque vulnerability. $J$ Nucl Cardiol. 2014;21:1112-1128.
2. Bäck M, Yurdagul A Jr, Tabas I, Oorni K, Kovanen PT. Inflammation and its resolution in atherosclerosis: mediators and therapeutic opportunities. Nat Rev Cardiol. 2019;16:389-406.

3. Libby P, Pasterkamp G, Crea F, Jang IK. Reassessing the mechanisms of acute coronary syndromes. Circ Res. 2019;124:150-160.

4. Tabas I, Bornfeldt KE. Intracellular and intercellular aspects of macrophage immunometabolism in atherosclerosis. Circ Res. 2020;126:1209-1227.

5. Chinetti-Gbaguidi G, Colin S, Staels B. Macrophage subsets in atherosclerosis. Nat Rev Cardiol. 2015;12:10-17.

6. O'Neill LA, Kishton RJ, Rathmell J. A guide to immunometabolism for immunologists. Nat Rev Immunol. 2016;16:553-565.

7. Aarup A, Pedersen TX, Junker N, et al. Hypoxia-inducible factor-1alpha expression in macrophages promotes development of atherosclerosis. Arterioscler Thromb Vasc Biol. 2016;36:1782-1790.

8. Sarrazy V, Viaud M, Westerterp M, et al. Disruption of Glut1 in hematopoietic stem cells prevents myelopoiesis and enhanced glucose flux in atheromatous plaques of apoE(-/-) mice. Circ Res. 2016;118:1062-1077.

9. Yu EPK, Reinhold J, Yu H, et al. Mitochondrial respiration is reduced in atherosclerosis, promoting necrotic core formation and reducing relative fibrous cap thickness. Arterioscler Thromb Vasc Biol. 2017;37:2322-2332.

10. Saito H, Kuroda S, Hirata K, et al. Validity of dual MRI and F-FDG PET imaging in predicting vulnerable and inflamed carotid plaque. Cerebrovasc Dis. 2013;35:370-377.

11. Rudd JH, Warburton EA, Fryer TD, et al. Imaging atherosclerotic plaque inflammation with $\left[{ }^{18} \mathrm{~F}\right]$-fluorodeoxyglucose positron emission tomography. Circulation. 2002;105:2708-2711.

12. Rudd JH, Myers KS, Bansilal S, et al. ${ }^{18}$ Fluorodeoxyglucose positron emission tomography imaging of atherosclerotic plaque inflammation is highly reproducible: implications for atherosclerosis therapy trials. $J \mathrm{Am}$ Coll Cardiol. 2007;50:892-896.

13. Figueroa AL, Abdelbaky A, Truong QA, et al. Measurement of arterial activity on routine FDG PET/CT images improves prediction of risk of future CV events. JACC Cardiovasc Imaging. 2013;6:1250-1259.

14. Marnane M, Merwick A, Sheehan OC, et al. Carotid plaque inflammation on ${ }^{18} \mathrm{~F}$ fluorodeoxyglucose positron emission tomography predicts early stroke recurrence. Ann Neurol. 2012;71:709-718.

15. Tawakol A, Fayad ZA, Mogg R, et al. Intensification of statin therapy results in a rapid reduction in atherosclerotic inflammation: results of a multicenter fluorodeoxyglucose-positron emission tomography/computed tomography feasibility study. J Am Coll Cardiol. 2013;62:909-917.

16. Zhao TX, Mallat Z. Targeting the immune system in atherosclerosis: JACC stateof-the-art review. J Am Coll Cardiol. 2019;73:1691-1706.

17. Al-Mashhadi RH, Tolbod LP, Bloch LO, et al. ${ }^{18}$ Fluorodeoxyglucose accumulation in arterial tissues determined by PET signal analysis. $\mathrm{J} \mathrm{Am} \mathrm{Coll} \mathrm{Cardiol.}$ 2019;74:1220-1232.

18. Guillermier C, Doherty SP, Whitney AG, et al. Imaging mass spectrometry reveals heterogeneity of proliferation and metabolism in atherosclerosis. JCI Insight. 2019;4:e128528.

19. Tavakoli S, Downs K, Short JD, et al. Characterization of macrophage polarization states using combined measurement of 2-deoxyglucose and glutamine accumulation: implications for imaging of atherosclerosis. Arterioscler Thromb Vasc Biol. 2017;37:1840-1848.

20. Tavakoli S, Short JD, Downs K, et al. Differential regulation of macrophage glucose metabolism by macrophage colony-stimulating factor and granulocytemacrophage colony-stimulating factor: implications for ${ }^{18}$ F-FDG PET imaging of vessel wall inflammation. Radiology. 2017;283:87-97.

21. Folco EJ, Sheikine Y, Rocha VZ, et al. Hypoxia but not inflammation augments glucose uptake in human macrophages: implications for imaging atherosclerosis with ${ }^{18}$ fluorine-labeled 2-deoxy-D-glucose positron emission tomography. $\mathrm{J} \mathrm{Am}$ Coll Cardiol. 2011;58:603-614.

22. Di Gioia M, Spreafico R, Springstead JR, et al. Endogenous oxidized phospholipids reprogram cellular metabolism and boost hyperinflammation. Nat Immunol. 2020;21:42-53.

23. Koelwyn GJ, Corr EM, Erbay E, Moore KJ. Regulation of macrophage immunometabolism in atherosclerosis. Nat Immunol. 2018;19:526-537.

24. Viola A, Munari F, Sanchez-Rodriguez R, Scolaro T, Castegna A. The metabolic signature of macrophage responses. Front Immunol. 2019;10:1462.

25. Cramer T, Yamanishi Y, Clausen BE, et al. HIF-1alpha is essential for myeloid cell-mediated inflammation. Cell. 2003;112:645-657.

26. van der Valk FM, Sluimer JC, Voo SA, et al. In vivo imaging of hypoxia in atherosclerotic plaques in humans. JACC Cardiovasc Imaging. 2015;8:1340-1341.

27. Joshi FR, Manavaki R, Fryer TD, et al. Vascular imaging with ${ }^{18} \mathrm{~F}$-fluorodeoxyglucose positron emission tomography is influenced by hypoxia. J Am Coll Cardiol. 2017;69:1873-1874. 
28. Nie X, Elvington A, Laforest R, et al. ${ }^{64} \mathrm{Cu}-\mathrm{ATSM}$ positron emission tomography/ magnetic resonance imaging of hypoxia in human atherosclerosis. Circ Cardiovasc Imaging. 2020;13:e009791.

29. Bose S, Ramesh V, Locasale JW. Acetate metabolism in physiology, cancer, and beyond. Trends Cell Biol. 2019;29:695-703.

30. Derlin T, Habermann CR, Lengyel Z, et al. Feasibility of ${ }^{11} \mathrm{C}$-acetate PET/CT for imaging of fatty acid synthesis in the atherosclerotic vessel wall. $\mathrm{J} \mathrm{Nucl} \mathrm{Med}$. 2011;52:1848-1854.

31. Demirdelen S, Mannes PZ, Aral AM, et al. Divergence of acetate uptake in proinflammatory and inflammation-resolving macrophages: implications for imaging atherosclerosis. J Nucl Cardiol. January 8, 2021 [Epub ahead of print].

32. Hedtke V, Bakovic M. Choline transport for phospholipid synthesis: an emerging role of choline transporter-like protein 1. Exp Biol Med (Maywood). 2019;244:655-662.

33. Snider SA, Margison KD, Ghorbani P, et al. Choline transport links macrophage phospholipid metabolism and inflammation. J Biol Chem. 2018;293:11600-11611.

34. Sanchez-Lopez E, Zhong Z, Stubelius A, et al. Choline uptake and metabolism modulate macrophage IL-1beta and IL-18 production. Cell Metab. 2019;29:1350-1362.e7.
35. Visentin M, van Rosmalen BV, Hiller C, et al. Impact of organic cation transporters (OCT-SLC22A) on differential diagnosis of intrahepatic lesions. Drug Metab Dispos. 2017;45:166-173

36. Bucerius J, Schmaljohann J, Bohm I, et al. Feasibility of ${ }^{18}$ F-fluoromethylcholine $\mathrm{PET} / \mathrm{CT}$ for imaging of vessel wall alterations in humans: first results. Eur J Nucl Med Mol Imaging. 2008;35:815-820.

37. Kato K, Schober O, Ikeda M, et al. Evaluation and comparison of ${ }^{11} \mathrm{C}$-choline uptake and calcification in aortic and common carotid arterial walls with combined PET/CT. Eur J Nucl Med Mol Imaging. 2009;36:1622-1628.

38. Förster S, Rominger A, Saam T, et al. ${ }^{18}$ F-fluoroethylcholine uptake in arterial vessel walls and cardiovascular risk factors: correlation in a PET-CT study. Nuklearmedizin. 2010;49:148-153.

39. Vöö S, Kwee RM, Sluimer JC, et al. Imaging intraplaque inflammation in carotid atherosclerosis with ${ }^{18} \mathrm{~F}$-fluorocholine positron emission tomography-computed tomography: prospective study on vulnerable atheroma with immunohistochemical validation. Circ Cardiovasc Imaging. 2016;9:e004467.

40. Jha AK, Huang SC, Sergushichev A, et al. Network integration of parallel metabolic and transcriptional data reveals metabolic modules that regulate macrophage polarization. Immunity. 2015;42:419-430. 